\title{
Fluctuation analysis of stellar x-ray binary systems
}

\author{
M. A. Moret, ${ }^{1,2}$ G. F. Zebende, ${ }^{1,2, *}$ E. Nogueira, Jr., ${ }^{3}$ and M. G. Pereira ${ }^{1}$ \\ ${ }^{1}$ Departamento de Física, Universidade Estadual de Feira de Santana, Km 3, BR116, Campus Universitário, \\ 44031-460 Feira de Santana, Bahia, Brazil \\ ${ }^{2}$ CEPPEV-Fundação Visconde de Cairu, Avenida Cardeal da Silva 14, Federação, 40226-900 Salvador, Bahia, Brazil \\ ${ }^{3}$ Instituto de Física, Universidade Federal da Bahia, Campus Universitário de Ondina, 40210-340 Salvador, Bahia, Brazil
}

(Received 29 March 2003; revised manuscript received 31 July 2003; published 13 October 2003)

\begin{abstract}
We study time series of x-ray sources of 129 stellar binary systems present in the public data collected by the instrument All Sky Monitor on board of the satellite Rossi X-Ray Timing Explorer. The light time series was analyzed by applying detrended fluctuation analysis to estimate the long-range power-law correlation exponents $\alpha$. The scaling exponent was calculated for all systems and its value indicated a signature of each kind of system, i.e., whether flare takes place (with $\alpha=1.22$ ) or not (with $\alpha=0.64$ ). As a consequence, our results may identify the stability of the systems from the scaling exponent $\alpha$ value, for instance, if $\alpha \sim 0.5$ (white noise) the system is stable and unstable when $\alpha \neq 0.5$ (long-range power-law correlation).
\end{abstract}

DOI: 10.1103/PhysRevE.68.041104

PACS number(s): 05.40.-a, 05.45.Tp, 97.80.-d

In recent years, there has been a growing evidence that many complex physical, economical, and biological systems manifest self-affinity characterized by long-range power-law correlations. In this context, the detrended fluctuation analysis (DFA) was recently proposed [1] to analyze long-range power-law correlations in nonstationary systems. One advantage of the DFA method is that it allows the long-range power-law correlations in signals with embedded polynomial trends that can mask the true correlations in the fluctuations of a noise signal. The DFA method has been applied to analyze DNA and its evolution [1-5], file editions in computer diskettes [6], economics [7-9], climate temperature behavior $[10,11]$, river flow and discharge $[12,13]$, and cardiac dynamics [14-17], among others. However, DFA has no longer been used in astrophysics, in which the effect of long-range correlation has been studied in simulations of self-gravitating systems [18], in the analysis of galaxy distribution [19] and in the characterization of self-similarity in solar active region [20]. Studies of x-ray binary systems using spectral and frequency residual analysis of gamma ray observations have been carried out, in a usual way, in Ref. [21,22].

In this paper we apply the DFA method to study the longterm time series of light fluctuations in the x-ray band produced in accretion power of stellar X-ray binary systems (XRB).The accretion power $\mathrm{X}$-ray binaries are an important class of astrophysical objects [23]. These stellar XRB contain either a neutron star or a black hole accreting material from a normal companion star. Approximately, 280 XRB have been identified from various X-ray surveys and optical identification programs [24,25]. The optical identification is necessary in order to establish the nature of the massdonating companion star, the geometry of the accretion flow, and the mass of the $\mathrm{x}$-ray source (the neutron star or the black hole) [26]. In addition, the overall luminosity, spectral shape, and time variability of the emission are influenced by the mass of the compact object and the mass accretion rate.

*Electronic address: zebende@uefs.br
The primary factors that determine the emission properties of an accretion compact object are: (a) the nature of the compact object, regarding it is a black hole or a neutron star; (b) the strength and geometry of its magnetic field, in the case of a neutron star; and (c) the geometry of the disk or spherical wind accretion flow from the companion star. These factors determine whether the emission region is the small magnetic polar cap of a neutron star, a hot accretion disk surrounding a black hole, a shock heated region in a spherical inflow, or the boundary layer separating the accretion disk from the neutron star.

The spectral type of the donor normal star determines the type of XRB, the mode of mass transfer, and the environment of the compact object. Usually XRB is classified into two main classes: Low mass X-ray binaries (LMXB) and high mass $\mathrm{X}$-ray binaries (HMXB), see squeme in Fig. 1. In the LMXB, a late type star does not have a natural wind strong enough to power the observed x-ray source. The efficient mass transfer will occur if the companion fills its critical gravitational potential Roche lobe. A stream of stellar material will be driven through the inner Lagrangian point, and will orbit the compact object. Viscous interactions cause this orbit to spiral, producing a large disk around the compact object [27]. In HMXB an early type star has an intense stellar wind, and the compact object, in a relatively close orbit, will capture a significant fraction of the wind, suffi-

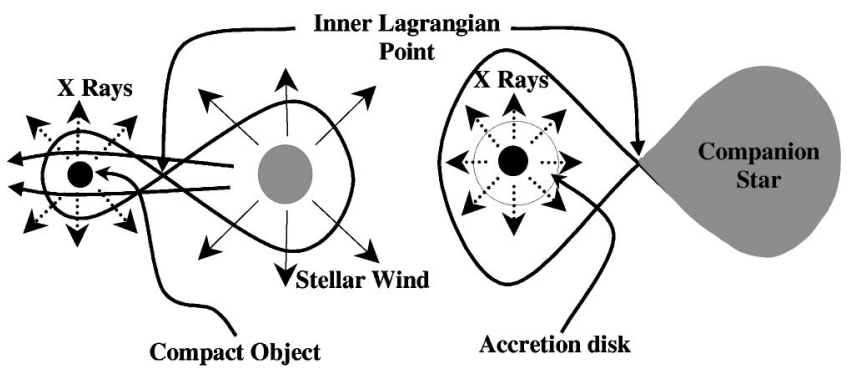

(a) HMXB

(b) LMXB

FIG. 1. X-ray binary system (XRB): (a) high mass companion stars (HMXB) and (b) low mass companion stars (LMXB). 
cient to power the $\mathrm{x}$-ray source [28]. In these mass systems Roche lobe overflow can also be a supplement to the mass transfer rate.

The light in the x-ray band produced in these systems is due to the accelerated stellar material in the innermost part of the accretion disk, the shock of material in the polar caps of the neutron star, and the explosive nuclear burn (bursts) on the neutron star surface. Instability in the accretion mass transfer can produce $\mathrm{x}$-ray transient outburst or flare in the accretion disk. Modulations are also observed in the $\mathrm{x}$-ray light due to the orbital movement of the XRB.

We have analyzed the data of 129 time series selected on public astronomic data obtained in the official All Sky Monitor (ASM) [34]. The ASM is an instrument on board of the scientific satellite Rossi X-Ray Timing Explorer (RXTE) to study the X-ray sky. The RXTE is operational since 1995, covering a spectral band of $\mathrm{x}$-ray photons corresponding to $1.5-12 \mathrm{keV}$ [29]. The main goal of the ASM is to alert observers to the appearance of transient x-ray emitters and a long-term monitoring of bright x-ray sources. The ASM consists of three scanning shadow cameras (SSCs) mounted on a motorized rotation drive. Each SSC contains a positionsensitive proportional counter that views the sky through a slit mask. Event data are normally compressed within the two ASM Event Analyzers in the Experiment Data System and relayed to the spacecraft for insertion in the telemetry stream. One ASM Event Analyzer accumulates histograms of counts binned as a function of position for each of the resistive anodes. These position histograms are accumulated in the series of 190's "dwells." During each dwell, the spacecraft maintains a fixed altitude and the ASM rotation drive, which is also controlled by this Event Analyzer, is not active so that the orientation of each SSC is fixed in relation to the sky. A position histogram thus contains the superposition of the mask shadows from each X-ray source in the field of view during a single dwell. The other ASM Event Analyzer produces count rates for both $\mathrm{x}$-ray and background events. The ASM collects useful data with a duty cycle of $\sim 40 \%$. This duty cycle, together with spacecraft maneuvers that are planned to carry out the observing program of the other RXTE instruments, produces a highly stochastic pattern of sky coverage with a randomly chosen source being scanned typically five to ten times per day.

The ASM data analysis proceeds by computing intensities for sources listed as active in a master catalog and then by searching for and locating additional sources. Source intensities are obtained from the solution of a linear least-squares fit of position histograms with model shadow patterns for each active source within the field of view and with patterns representing non-X-ray and diffuse x-ray backgrounds [30]. The fit solution also yields estimates of the uncertainties of the derived intensities; these uncertainties are based purely on the photon counting statistics predicted by the best fit model. Fitted intensities are normalized to on-axis count rates in SSC 1. This requires two corrections, the first of which must be applied because the position histogram models computed for unit count rate sources do not yet fully take into account the loss of effective area for sources at large elevations in the field of view. At present, this correction factor has been empirically determined from observations of the Crab Nebula. The second correction accounts for absorption in the polyimide coating on the windows of SSC 2 and SSC 3, so that the results from all three SSCs are consistent. Source intensities and errors (and estimated adjustments to the pointing direction) are reported from the fit with the minimum value of the reduced qui square statistic. The fit residuals are examined via a cross-correlation technique for evidence of sources beyond those from the active list. When such a source is detected, the cross-correlation function is used to estimate its celestial location.

In order to analyze the XRB time series, we briefly present an introduction to the DFA method [1,31], which involves the following steps:

(1) Consider a correlated signal intensity, $u(i)$ (counts/s), where $i=1, \ldots, N_{\max }$ and $N_{\max }$ is the total number of days in the ASM. We integrate the signal $u(i)$ and obtain $y(k)$ $=\sum_{i=1}^{k} u(i)-\langle u\rangle$, where $\langle u\rangle$ stands for the average value of $u$;

(2) The integrated signal $y(k)$ is divided into boxes of equal length $n$.

(3) For each $n$-size box, we fit $y(k)$, using a polynomial function of order $l$, which represents the trend in the box. The $y$ coordinate of the fitting line in each box is denoted by $y_{n}(k)$, since we use a polynomial fitting of order $l$, we denote the algorithm as DFA- $l$;

(4) The integrated signal $y(k)$ is detrended by subtracting the local trend $y_{n}(k)$ in each box (of length $n$ ).

(5) For a given $n$-size box, the root-mean-square fluctuation, $F(n)$, for this integrated and detrended signal is given by

$$
F(n)=\sqrt{\frac{1}{N_{\max }} \sum_{k=1}^{N_{\max }}\left[y(k)-y_{n}(k)\right]^{2}} .
$$

(6) The above computation is repeated for a broad range of scales ( $n$-size box) to provide a relationship between $F(n)$ and the box size $n$, characterized for a power-law $F(n) \sim n^{\alpha}$. In this way, $\alpha$ is the scaling exponent, a selfaffinity parameter representing the long-range power-law correlation properties of the signal, such that if $\alpha=0.5$ the signal is uncorrelated, if $\alpha<0.5$ the correlation in the signal is antipersistent, and if $\alpha>0.5$ the correlation in the signal is persistent.

Note that for antipersistent signals, the scaling exponent obtained from the DFA method overestimates the true correlations at small scales. To avoid this problem, one needs first to integrate the original anticorrelated signal and then apply the DFA method. The correct scaling exponent can thus be obtained from the relation between $n$ and $F(n) / n$ [instead of $F(n)]$. When the signal had an anticorrelated behavior we evaluated $\alpha$ using this methodology [32]. The DFA analysis was carried out over light curves of XRB observed during $\sim 2000$ days, and all the systems show a power-law with one characteristic long-range correlation exponent $\alpha$.

The x-ray intensity presented in the light curves of the $\mathrm{XRB}$ is due to mass transfer of these systems, Fig. 2 upper inset. For a stationary accretion disk, it is expected a corre- 


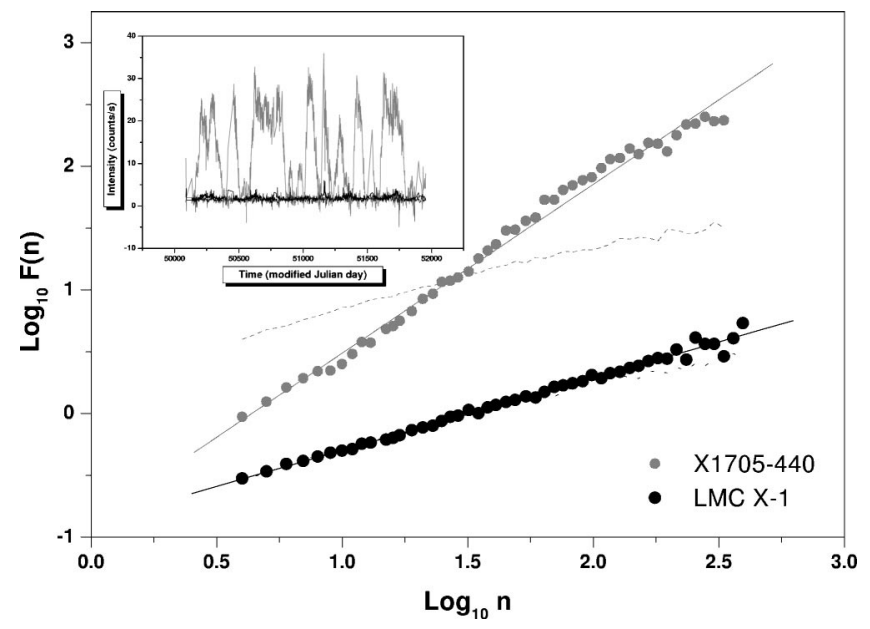

FIG. 2. Two examples of XRB, flare X1705-440 (gray) and no flare LMC X-1 (black). The upper inset represents the intensity of these light curves. The scaling exponent $\alpha$ for these two cases are 1.36 (gray) and 0.58 (black). The dashed lines represent the surrogate time series from the original data.

lated signal dominated by the random variations of bright. However, flares are observed in several systems. We recall that flares are abrupt increases of the accretion mass and produce an increasing bright of the $\mathrm{x}$-ray band, indicating the presence of disk instabilities. On the other hand, variations due to periodicity do not change the light intensity observed in our analysis of the time series. Using DFA we detrend flares and periodicity in the time series and analyze the influence of fluctuations. Figure 2 depicts the behavior of the LMCX-1 and X1705-440 systems. In this figure we observe that the scaling exponent $\alpha$ tends to two different values $\alpha_{L M C X-1}=0.58$ and $\alpha_{X 1705-440}=1.36$. To show that trends were removed by DFA in the time series, the original data was time random shuffled producing a surrogate time series [33], where the $\alpha$ exponents for these new sequences, as expected, is $\alpha_{L M C X-1}^{m i x}=0.50 \pm 0.01$ and $\alpha_{X 1705-440}^{m i x}=0.48$ \pm 0.01 (dashed lines Fig. 2). In accordance with [1,31], the smallest box width $(5)$ and largest box width $\left(\sim N_{\max } / 4\right)$ was used.

The exponent $\alpha$ for all original time series, using a polynomial fitting of the order of 1, gave rise to the histograms showed in Fig. 3(a). An interesting view of these histograms is given by analyzing XRB, when we set apart the cases without flare, Fig. 3(b), from those with flare, Fig. 3(c). In Fig. 3(b) we observe the mean $\alpha=0.64 \pm 0.12$ with a normal distribution, when the accretion mass transfer tend to a stable behavior $(\alpha=0.5)$. On the other hand, if instability in accretion mass transfer occurs producing flares, Fig. 3(c), the mean scaling exponent $\alpha$ is $1.22 \pm 0.16$. Therefore, there is a strongly correlated signal, meaning that mass transfer in ac-
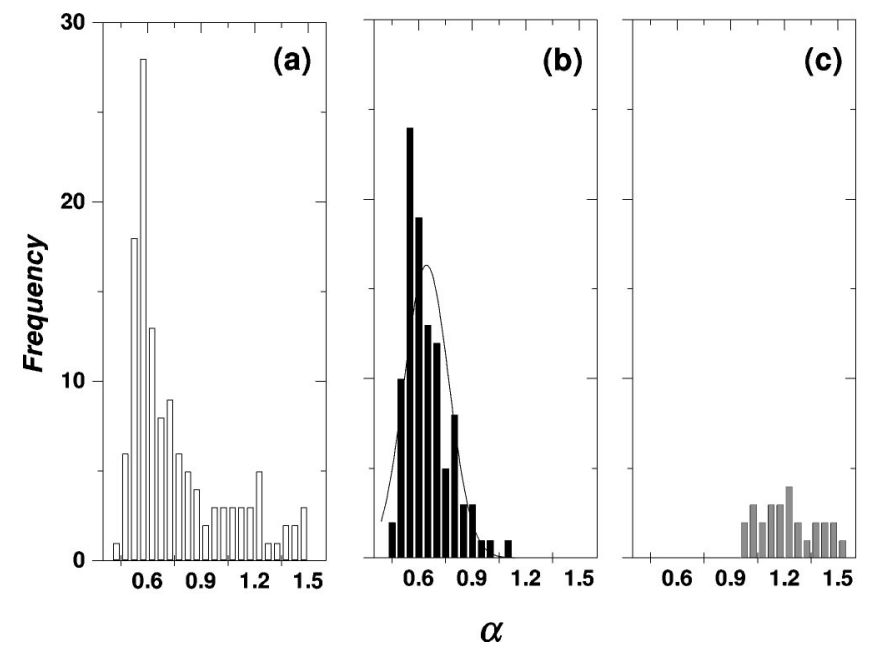

FIG. 3. (a) Histogram for the 129 scaling exponent $\alpha$ studied in this paper. (b) Histogram for the XRB with a stable accretion mass transfer. (c) Histogram for the XRB with flare occurrence.

cretion disk is a cooperative effect, i.e., when the mass accretion on the disk increases (decreases) there is a high tendency that mass flux continues to increase (decreasing). Beyond that, we observe that for both late and early type stars the expected value of the scaling exponent $\alpha$ tends to a persistent correlated value, showing that these systems have the mass accretion from the accretion disk as a cooperative flux, i.e., if 1 day the mass flux increases (decreases), there is a high tendency that the next day it remains the same.

In summary, we have studied the light curves in different classes of the XRB (LMXB and HMXB). Such systems reflect different accretion mass rate from the mass-donating companion star. From the results we observe that if instability in accretion mass transfer occurs (flare) the correlation is more persistent than systems where the accretion mass transfer is stable. This suggests that the accretion mass of the disk is due to the cooperative and persistent mass flux, such that occurring flare, the long-range correlation is greater than the other type (without flare occurrence). The behavior of the scaling exponent $\alpha$ allows us to measure the existence of this instability in this type of stellar systems. The present contribution shows that the scaling exponent $\alpha$ was calculated for XRB observed by ASM and its value indicated a signature of the systems, whether the flare occurs (with average estimate of the $\alpha=1.22$ ) or not ( $\alpha=0.64$ ). Therefore, characterizing XRB by using a simple methodology based on the scaling exponent $\alpha$, we may identify if the system is stable or unstable.

M.A.M. and G.F.Z. wish to thank Paulo Murilo Castro de Oliveira for discussions. We are indebted to Ademir Eugenio Santana for a critical reading of the manuscript. This work was supported in part by CNPq and FAPESB.
[1] C.-K. Peng, S.V. Buldyrev, S. Havlin, M. Simons, H.E. Stanley, and A.L. Goldberger, Phys. Rev. E 49, 1685 (1994).

[2] C.-K. Peng, S.V. Buldyrev, A.L. Goldberger, S. Havlin, F. Sciortino, M. Simons, and H.E. Stanley, Nature (London) 356,
168 (1992).

[3] C.-K. Peng, S.V. Buldyrev, A.L. Goldberger, S. Havlin, M. Simons, and H.E. Stanley, Phys. Rev. E 47, 3730 (1993).

[4] V. Buldyrev, A.L. Goldberger, S. Havlin, C.-K. Peng, H.E. 
Stanley, and M. Simons, Biophys. J. 65, 2673 (1993).

[5] H.E. Stanley, S.V. Buldyrev, A.L. Goldberger, S. Havlin, C.-K. Peng, and M. Simons, Physica A 273, 1 (1999).

[6] G.F. Zebende, P.M.C. de Oliveira, and T.J.P. Penna, Phys. Rev. E 57, 3311 (1998).

[7] Y. Liu, P. Gopikrishnan, P. Cizeau, M. Meyer, C.-K. Peng, and H.E. Stanley, Phys. Rev. E 60, 1390 (1999).

[8] R.N. Mantegna and H.E. Stantey, Nature (London) 367, 46 (1995)

[9] R.N. Mantegna and H.E. Stantey, An Introduction to Econophysics: Correlations and Complexity in Finance (Cambridge University Press, Cambridge, 1999).

[10] E. Koscielny-Bunde, A. Bunde, S. Havlin, H.E. Roman, Y. Goldreich, and H.J. Schellnhuber, Phys. Rev. Lett. 81, 729 (1998).

[11] P. Talkner and R.O. Weber, Phys. Rev. E 62, 150 (2000).

[12] A. Montanari, R. Rosso, and M.S. Taqqu, Water Resour. Res. 36, 1249 (2000).

[13] C. Matsoukas, S. Islam, and I. Rodriguez-Iturbe, J. Geophys. Res., [Atmos.] 105, 29165 (2000).

[14] P.Ch. Ivanov, M.G. Rosenblum, C.-K. Peng, J.E. Mietus, S. Havlin, H.E. Stanley, and A.L. Goldberger, Nature (London) 383, 323 (1996).

[15] P.Ch. Ivanov, L.A. Nunes Amaral, A.L. Goldberger, S. Havlin, M.G. Rosenblum, Z.R. Struzik, and H.E. Stanley, Nature (London) 399, 461 (1999).

[16] T.H. Makikallio, J. Koistinen, L. Jordaens, M.P. Tulppo, N. Wood, B. Golosarsky, C.-K. Peng, A.L. Goldberger, and H.V. Huikuri, Am. J. Cardiol. 83, 880 (1999).

[17] A. Bunde, S. Havlin, J.W. Kantelhardt, T. Penzel, J.H. Peter, and K. Voigt, Phys. Rev. Lett. 85, 3736 (2000).
[18] D. Huber and D. Pfenniger, Astron. Astrophys. 386, 359 (2002).

[19] H. di Nella, M. Montuori, G. Paturel, L. Pietronero, and F. Sylos Labini, Astron. Astrophys. 308, L33 (1996).

[20] R.R. Rosa, H.O. Vats, F.M. Ramos, A. Zanandrea, C. Rodrigues Neto, F.C.R. Fernandes, M.J.A. Bolzan, E.L. Rempel, R.C. Brito, N.L. Vijaykumar, and H.S. Sawant, Adv. Space Res. 29, 463 (2002).

[21] M.G. Pereira, J. Braga, and F. Jablonski, Astrophys. J. Lett. 526, L105 (1999).

[22] F. Jablonski, M.G. Pereira, J. Braga, and C. Gneiding, Astrophys. J. Lett. 482, L171 (1997).

[23] N.E. White, Astron. Astrophys. Rev. 1, 85 (1989).

[24] Q.Z. Liu, J. van Paradijs, and E.P.J. van den Heuvel, Astron. Astrophys. 368, 1021 (2001).

[25] Q.Z. Liu, J. van Paradijs, and E.P.J. van den Heuvel, Astron. Astrophys., Suppl. Ser. 147, 25 (2000).

[26] J. Frank, A.R. King, and D.J. Reine, Accretion Power in Astrophysics, 2nd ed. (Cambridge University Press, Cambridge, 1992).

[27] S.H. Lubow and F.H. Shu, Astrophys. J. 198, 383 (1975).

[28] J.E. Pringle and M.J. Rees, Astron. Astrophys. 21, 1 (1972).

[29] A.M. Levine, H. Bradt, W. Cui, J.G. Jernigan, E.H. Morgan, R. Remillard, R.E. Shirey, and D.A. Smith, Astrophys. J. 469, L33 (1996).

[30] J.P. Doty, Proc. SPIE 982, 164 (1988).

[31] http://www.physionet.org/physiotools/dfa/dfa.c

[32] Z. Chen, P.C. Ivanov, K. Hu, and H.E. Stanley, Phys. Rev. E 65, 041107 (2002).

[33] T. Schereiber and A. Schmitz, Physica D 142, 346 (2000).

[34] http://xte.mit.edu/ASM_lc.html 